\title{
Human Parasitic Diseases: A Diagnostic Atlas
}

Lawrence R. Ash and Thomas C. Orihel, American Society for Clinical Pathology Press, USA (Printed in Hong Kong, 2020), 672 p, ISBN 0-89189-6777.

The atlas book Human Parasitic Diseases: A Diagnostic Atlas consists of six large sections (Protozoa, Helminths \& Nematodes, Trematodes, Cestodes, Other Parasites and Non-Parasites, and Arthropods) and two appendix parts (Quick Keys to Identification and Index). I found that this atlas book contains comprehensive collections of morphological, biological, clinico-pathological, and diagnostic details of important humanparasitic protozoans and helminths occurring all around the world. The front part of each section is composed of the basic and essential knowledge on each species of parasite or group of parasites. A vast collection of excellent figures of parasites and involved host tissues is exhibited in the latter part of each section.

I am surprised to see that the taxonomy and biology of the parasites described in this book have been most freshly updated, and numerous newest knowledge and information in the field of medical and clinical parasitology have been gathered and introduced. I also see that the book contains quick keys to the identification of human intestinal protozoa in stained smears, helminth eggs in feces and body fluids, microfilariae found in humans, and filariform larvae in fecal cultures. That appears to be practically useful is a chapter on artifacts, including large-sized objects, pseudoparasites, cellular elements, and tissue elements which are frequently confused with parasites.

The quality of figures is superb, and the explanations using numbers and symbols are excellent. Malaria parasites in blood films are shown page-wide, and numerous small inset figures between the text are very beautiful and artistic. Keys to identification of various protozoa and helminths in feces, body fluids, blood, and fecal cultures are excellent and practically useful.

Nowadays, training of young experts in the field of medical parasitology and tropical medicine is urgently necessitated in order to provide proper diagnosis followed by adequate management of parasite-infected patients. This book undoubtedly will be highly helpful for this purpose. In addition, medical doctors, parasitology researchers and professors, and laboratory personnel who need refreshing and continuing education, will also find this book most useful.

Jong-Yil Chai (cjy@snu.ac.kr)

Professor Emeritus, Department of Tropical Medicine and Parasitology, Seoul National University College of Medicine, Seoul 03080, Korea President, Korea Association of Health Promotion, Seoul 07649, Korea 
